Proc. Indian Acad. Sci. (Chem. Sci.), Vol. 105, No. 6, December 1993, pp. 505-512.

(C) Printed in India.

\title{
Photoinduced charge transfer processes in ultrasmall semiconductor clusters. Photophysical properties of CdS clusters in Nafion membrane
}

\author{
K R GOPIDAS ${ }^{\dagger}$ and PRASHANT V KAMAT* \\ Radiation Laboratory, University of Notre Dame, Notre Dame, Indiana 46556, USA \\ ${ }^{\dagger}$ Present address: Regional Research Laboratory, Trivandrum 695019, India
}

\begin{abstract}
The photophysical properties of quantized CdS clusters in a perfluorosulfonate polymer (Nafion) film have been investigated by time-resolved emission spectroscopy. The ultrasmall CdS clusters were prepared by exposing $\mathrm{Cd}^{2+}$-exchanged polymer film to $\mathrm{H}_{2} \mathrm{~S}$. Size-dependent absorption and emission properties were observed during the growth of these clusters. The emission decay is multiexponential with lifetimes ranging from 0.85 to $480 \mathrm{~ns}$.
\end{abstract}

Keywords. Semiconductor clusters; CdS; size quantization effect; Nafion; emission lifetimes.

\section{Introduction}

Considerable attention has been given in recent years to the application of semiconductor colloids, powders and films for conversion of solar energy into electricity and chemical energy (Bard 1982; Kalyanasundaran et al 1986; Henglein 1988, 1989; Memming 1988; Grätzel 1989; Kamat and Dimitrijevic 1990; Kamat 1991, 1993). Of particular interest are the quantized semiconductor colloids which exhibit hybrid molecular solid state properties (Brus 1986; Henglein 1988, 1989; Bawendi et al 1990; Steigerwald and Brus 1990; Wang and Herron 1991; Kamat and Meisel 1993). These ultrasmall colloidal particles contain a high density of defect sites, usually at the semiconductor surface, and the nature of these defect sites depends strongly on the method of chemical synthesis. Upon optical excitation, the free carriers are rapidly trapped at the defect sites and these trapped charge carriers further undergo radiative and nonradiative recombination.

The mechanistic and kinetic details of the charge transfer processes in metal oxide $\left(\mathrm{TiO}_{2}, \mathrm{ZnO}\right)$ and metal chalcogenides (CdS, $\left.\mathrm{CdSe}\right)$ have been presented in our earlier studies (see, for example, Kamat and Dimitrijevic 1990, Kamat 1991, 1993, for detailed reviews on this topic). Both transient absorption and emission spectroscopy techniques have been employed to characterize the primary photophysical and photochemical events that occur in the picosecond-millisecond time domain.

Efforts have also been made to prepare ultrasmall semiconductor particles in various heterogeneous environments (Fendler 1985, 1987; Wilner and Wilner 1988; Fox 1991). Microencagement of semiconductor particles in an organized medium controls not only the morphology but also the photocatalytic properties of the semiconductors. For example, metal chalcogenide clusters can easily be synthesized

\footnotetext{
* For correspondence
} 
in a polymer film (Meisner et al 1983; Kuczynski et al 1984; Wang and Mahler 1987; Mahler 1988; Honda et al 1988; Nosaka et al 1989; Dalas et al 1990; Gopidas and Kamat 1990; Miyoshi et al 1990; Smotkin et al 1990; Misawa et al 1991; Yoneyama 1991). Such preparations provide isolated small semiconductor particles which are stabilized in an organized medium. In most of these studies, CdS has been the popular choice since it can be prepared easily by chemical precipitation and characterized readily by absorption and emission spectra. The photophysical properties of CdS clusters prepared in Nafion membrane are presented in this paper.

\section{Experimental section}

\subsection{Materials}

Nafion 117 in $\mathrm{H}^{+}$form was obtained from Aldrich and $\mathrm{H}_{2} \mathrm{~S}$ gas was obtained from Matheson Gas Products. All other chemicals were analytical reagents and were used as supplied.

\subsection{Sample preparation}

The Nafion film was extracted with methanol for $4-5 \mathrm{~h}$ and was dried in an oven at $60^{\circ} \mathrm{C}$ for $24 \mathrm{~h}$. The sodium-exchanged form of the Nafion was prepared by soaking the film in an aqueous solution of $1 \mathrm{M} \mathrm{NaOH}$ for $24 \mathrm{~h}$ and then washing thoroughly with deionized water. The film was then dried in the oven for $24 \mathrm{~h}$. The optically transparent Nafion film was then cut into $0.5 \times 4 \mathrm{~cm}$ pieces so that the film could conveniently be introduced into a $2 \mathrm{~mm}$ thick optical cell.

$\mathrm{Cd}^{2+}$ ions in the Nafion film were exchanged by immersing $\mathrm{Na}^{+}-\mathrm{Nafion}$ in a $\mathrm{CdI}_{2}$ solution $\left(100 \mathrm{ml}\right.$ of $\left.10^{-5}-10^{-3} \mathrm{M}\right)$ for $30 \mathrm{~min}$ to $3 \mathrm{~h}$. The film was then thoroughly washed with deionized water and dried in an oven at $60^{\circ} \mathrm{C}$ for $24 \mathrm{~h}$. A single piece $(0.5 \times 4 \mathrm{~cm})$ of $\mathrm{Cd}^{2+}$-exchanged Nafion film was introduced in an optical cell $(2 \mathrm{~mm}$ thick). The cell was closed with a rubber septum and flushed with a stream of argon for $30 \mathrm{~min}$ to rẹmove adsorbed $\mathrm{O}_{2}$ from the film. About $10 \mathrm{ml}$ of $\mathrm{H}_{2} \mathrm{~S}$ gas was then injected into the cell for initiating CdS formation. The reaction could be arrested at any stage by quick degassing. The yellow coloration of the film confirmed the formation of $\mathrm{CdS}$ particles. Care was taken to exclude $\mathrm{O}_{2}$ from the cell. As a precautionary measure all the Nafion films containing CdS were stored in an argon atmosphere.

\subsection{Optical measurements}

The absorption spectra were recorded with a Perkin-Elmer 3840 diode-array spectrophotometer. The emission spectra were recorded with an SLM S-8000 photon-counting spectrofluorometer in a front-face configuration. Emission lifetime measurements were performed by the time-correlated single-photon counting technique using an apparatus that has been described elsewhere (Federici et al 1985). The excitation source was a mode-locked, $Q$-switched Quantronix $416 \mathrm{Nd}$ : YAG laser which provided $80 \mathrm{ps}$ pulses of $355 \mathrm{~nm}$ light with a frequency of $5 \mathrm{kHz}$ and an integrated power of $10 \mathrm{~mW}$.

Time-resolved emission spectra were recorded from laser flash photolysis experiments using $337 \mathrm{~nm}$ laser pulses from a PRA LN1000 nitrogen laser system 
(pulse width $0.5 \mathrm{~ns}$ ). The details of the experimental arrangement can be found elsewhere (Nagarajan and Fessenden 1985). All the experiments were done at room temperature $\left(23^{\circ} \mathrm{C}\right)$.

\section{Results and discussion}

\subsection{Formation of $C d S$ clusters in the Nafion film and size quantization effects}

The CdS clusters within the polymer matrix could be synthesized by exposing the $\mathrm{Cd}^{2+}$-doped Nafion films to an atmosphere of $\mathrm{H}_{2} \mathrm{~S}$. The absorption spectra recorded following the exposure of $\mathrm{Cd}^{2+} /$ Nafion to $\mathrm{H}_{2} \mathrm{~S}$ are shown in figure 1 . The color of the film slowly changed from colorless to yellow as the $\mathrm{H}_{2} \mathrm{~S}$ exposure time was increased. This is clearly evident from the increased absorption in the spectra recorded at longer times (figure 1). In the bulk form, CdS is deep yellow in color $\left(E_{g}=2.4 \mathrm{eV}\right.$ ) with an onset absorption around $520 \mathrm{~nm}$. However, in smaller diameter particles $(<50 \AA)$, the absorption shifts to the blue as the effective bandgap increases. The morphology of the Nafion polymer is such that it controls the clustering of CdS molecules (Dp 15-150 $)$ within the hydrocarbon network.

Semiconductor particles which exhibit size-dependent optical and electronic properties are termed quantized (or Q-) particles or nanoclusters (Meisner et al 1983; Kuczynski et al 1984; Wang and Mahler 1987; Honda et al 1988; Mahler 1988; Nosaka et al 1989; Dalas et al 1990; Gopidas and Kamat 1990; Miyoshi et al 1990; Smotkin et al 1990; Misawa et al 1991; Yoneyama 1991). These ultrasmall semiconductor particles are molecular clusters in which complete electron delocalization has not yet occurred. Quantization in these ultrafine semiconductor particles arises from the confinements of charge carriers with potential wells of small dimensions (less than the DeBroglie wavelength of the electrons and holes). Under these conditions the energy levels available for electrons and holes in the conduction and valence bands become discrete. The absorption spectra recorded in figure 1 show the transition from small molecular clusters to bulk aggregates. In addition to its very large effect

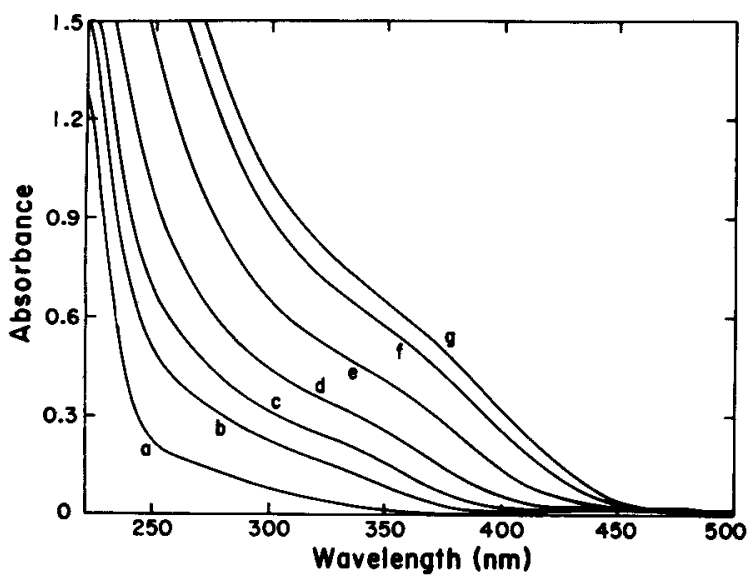

Figure 1. Absorption spectra of CdS clusters in Nafion film. The spectra were recorded at various time intervals following the exposure of the $\mathrm{Cd}^{2+}$-doped (1 mmole) Nafion film to $\mathrm{H}_{2} \mathrm{~S}$ for 0 (a), 5 (b), 10 (c), 20 (d), 45 (e), 90 (f), and $120 \mathrm{~min}(\mathrm{~g})$. 
on optical properties, size quantization also leads to enhanced redox activity of the photogenerated charge carriers.

The slow rate of CdS formation in dry Nafion film facilitates control of particle size by limiting the time of $\mathrm{H}_{2} \mathrm{~S}$ exposure. For example, it was possible to stop the growth of CdS clusters by quickly degassing the sample cell and storing the film in an inert $\left(\mathrm{N}_{2}\right.$ or $\left.\mathrm{Ar}\right)$ atmosphere. Similar control of particle size has also been reported for CdS (Nosaka et al 1989; Smotkin et al 1990; Misawa et al 1991), PbS (Mahler 1988) and CdSe (Gopidas and Kamat 1990; Yoneyama 1991) in polymer films. Another approach to control the particle size is to decrease the concentration of $\mathrm{Cd}^{2+}$ in the polymer film. By decreasing the concentration of $\mathrm{Cd}^{2+}$ from $10^{-4}$ moles to $10^{-6}$ moles, it is possible to grow Q-size CdS clusters with long time $\mathrm{H}_{2} \mathrm{~S}$ exposure. By diluting $\mathrm{Cd}^{2+}$ concentration in Nafion with inert cations such as $\mathrm{Ca}^{2+}$, it is also possible to control the size of CdS and CdSe clusters (Smotkin et al 1990).

\subsection{Emission spectra of CdS clusters in Nafion}

Emission spectra recorded during the growth of CdS crystallites are shown in figure 2. The blank film (spectrum $a$, recorded before the $\mathrm{H}_{2} \mathrm{~S}$ exposure) exhibits relatively small emission below $400 \mathrm{~nm}$. This emission, which arises as a result of some organic impurities imbedded in the Nafion film, does not interfere with the measurement of $\mathrm{CdS}$ emission. Once the $\mathrm{Cd}^{2+}$ doped Nafion film is exposed to $\mathrm{H}_{2} \mathrm{~S}$ a new emission band arises as CdS clusters are formed within the polymer matrix. As shown earlier, this emission mainly arises from the sulfur vacancies at the CdS crystallites. With increasing $\mathrm{H}_{2} \mathrm{~S}$ exposure time an increase in the $\mathrm{CdS}$ emission is seen. In the initial stages (spectra $a$ in figure 2), the growth in CdS clusters leads to an increased

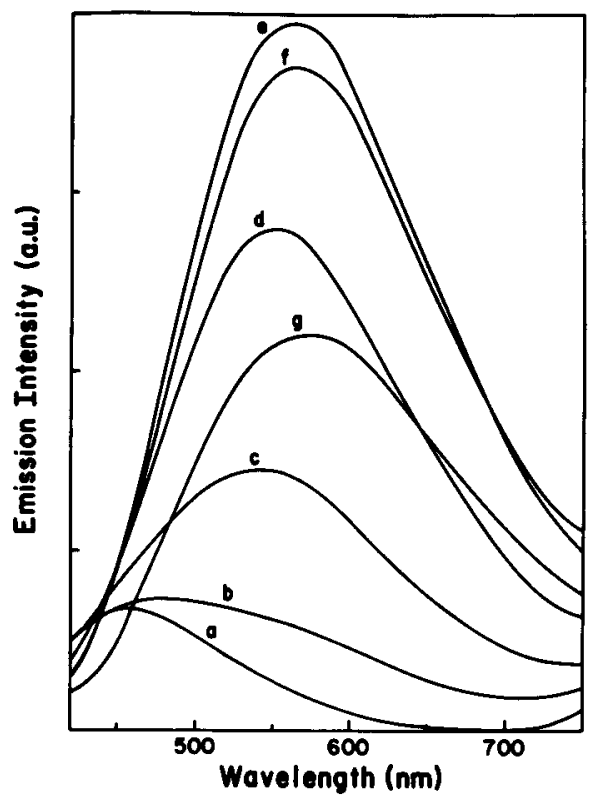

Figure 2. Emission spectra of CdS clusters in Nafion film. The spectra were recorded at various time intervals following the exposure of the $\mathrm{Cd}^{2+}$ doped ( 1 mmole) Nafion film to $\mathrm{H}_{2} \mathrm{~S}$ for 0 (a), 8 (b), 15 (c), 22 (d), 60 (e), 90 (f), and $180 \mathrm{~min}(\mathrm{~g})$. 
absorption at the excitation wavelength, which in turn leads to the enhancement of the emission yield. However, at longer times (spectra $f$ and $g$ in figure 2 ) a decrease in the emission yield is observed. This shows that once the CdS clusters are fully grown the efficiency of radiative recombination process decreases. Decreased surface area and saturation of sulfur vacancies in larger crystallites is likely to affect the radiative recombination in the larger clusters.

Another interesting feature is the shift in the emission maximum of the CdS clusters in the Nafion film. When the crystallites are small, the emission maximum is centered around $480 \mathrm{~nm}$. But as the CdS cluster grows, the emission band shifts to the red. In a fully grown cluster, the emission maximum is observed at $580 \mathrm{~nm}$. The blue shift in the emission maximum of smaller CdS clusters is parallel to the shift observed in the absorption edge (figure 1) and attributed to the size quantization effects. Thus, one can utilize the emission properties to probe the size quantization of $\mathrm{CdS}$ clusters in the polymer films.

\subsection{Emission lifetimes}

It has been shown earlier that the emission decay of semiconductor clusters provides important information regarding kinetic and mechanistic details of charge carrier recombination. A typical decay profile of CdS emission at $470 \mathrm{~nm}$ is shown in figure 3 . Such a nonexponential decay is attributed to the distribution of excited states at various trapping sites that emit with different lifetimes. This multiexponential decay was fit by a nonlinear least squares procedure to the three component decay law as given by (James et al 1985),

$$
F(t)=a_{1} \exp \left(-t / \tau_{1}\right)+a_{2} \exp \left(-t / \tau_{2}\right)+a_{3} \exp \left(-t / \tau_{3}\right)
$$

The lifetimes analyzed from its decay kinetics are summarized in table 1 . These range from 0.85 to $480 \mu \mathrm{s}$. The shorter-lived states were found to emit at higher energies.

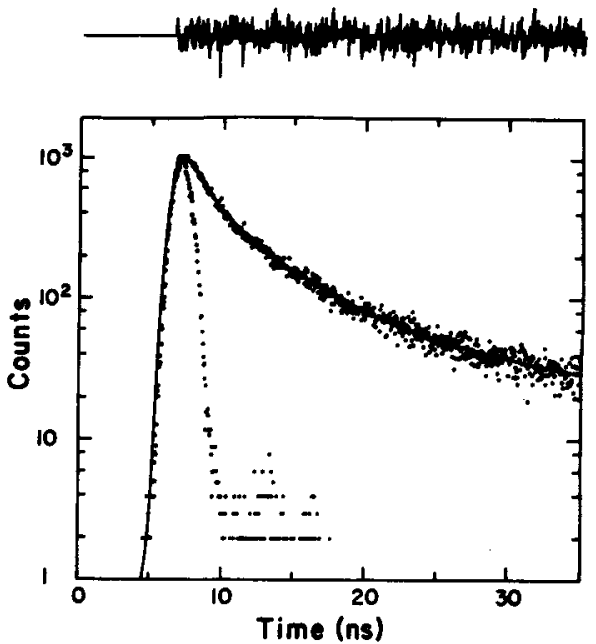

Figure 3. Emission decay profile of CdS clusters in Nafion monitored at $470 \mathrm{~nm}$. The analysis was carried out by fitting it to the three exponential decay kinetics, (1), using the parameters: $a_{1}=0.037, \tau_{1}=1.03 \mathrm{~ns}, a_{2}=0.019, \tau_{2}=4.29 \mathrm{~ns}, a_{3}=0.0029$ and $\tau_{3}=23.73 \mathrm{~ns}$. The profile of the laser pulse is also shown for comparison. 
Table 1. Emission lifetimes of CdS clusters in Nafion.

\begin{tabular}{lrrrr}
\hline Emission & \multicolumn{5}{c}{ Emission lifetimes" (ns) } \\
\cline { 2 - 5 } $\begin{array}{l}\text { wavelength } \\
\text { (nm) }\end{array}$ & \multicolumn{1}{c}{$\tau_{1}$} & \multicolumn{1}{c}{$\tau_{2}$} & \multicolumn{1}{c}{$\tau_{3}$} & $\langle\tau\rangle^{b}$ \\
\hline 400 & 0.85 & 3.32 & 14.73 & 5.61 \\
470 & 1.03 & 4.29 & 23.73 & 10.75 \\
580 & 14.80 & 111.51 & 379.75 & 179.10 \\
680 & 18.77 & 124.31 & 480.00 & 283.70 \\
\hline
\end{tabular}

- Lifetimes were analyzed by fitting the decay to three exponential decay kinetics

'The average lifetime $\langle\tau\rangle$ was calculated based on the expression of James et al (1985): $\langle\tau\rangle=\Sigma a_{i} \tau_{i}^{2} / \Sigma a_{i} \tau_{i}$

Similar wavelength-dependent emission lifetimes have been observed for $\mathrm{ZnO}, \mathrm{ZnS}$, CdS and CdSe colloids (see, for example, Kamat and Dimitrijević 1980, Kamat 1991, 1993). The wavelength dependence of the emission lifetime arises from the contribution of the Coulombic energy of interaction of the electron-hole pair to the total energy of the emitted photon. A more detailed analysis of these lifetimes in CdS clusters has been carried out by Chestnoy (Chestnoy et al 1986).

\subsection{Time-resolved emission spectra}

The contribution of various emitting centers which exhibit different emission lifetimes was further probed by recording time-resolved emission spectra. The CdS-doped Nafion film was excited with $337 \mathrm{~nm}$ laser pulses from an $\mathrm{N}_{2}$ laser (pulse width $0.5 \mathrm{~ns}$ ) and the emission spectra were recorded at different time intervals (figure 4). These spectra were normalized for the photomultiplier response. A decrease in the emission yield as well as a red-shift in the maximum was seen with increasing time. The spectrum recorded immediately after the pulse shows a maximum around $440 \mathrm{~nm}$, while the spectrum recorded $52 \mathrm{~ns}$ after the laser pulse has an emission maximum at $480 \mathrm{~nm}$. Similarity in the shape of the emission band in all these spectra suggests that the same surface vacancy (sulfur vacancy) is responsible for all the emission spectra recorded in figure 4 . The steady state emission spectrum at $77 \mathrm{~K}$ is also shown for comparison. This steady state emission band is considerably red-shifted and exhibits very little emission at higher energy (below $420 \mathrm{~nm}$ ). This further supports the earlier proposal (Chestnoy et al 1986) that close pairs with small separating distances emit faster and at higher energy than distant pairs for a fixed particle diameter.

\subsection{Possible applications of CdS films in solar energy conversion}

It has been shown earlier that CdS clusters imbedded in the polymer film are an excellent choice for the photoelectrolysis of water (Krishnan et al 1983; Meisner et al 1983; Finlayson et al 1988). Relatively large quantities of $\mathrm{H}_{2}$ generation were produced in the presence of an electron donor such as $\mathrm{S}^{2-}$. It should also be possible to utilize such thin semiconductor particulate films in photoelectrochemical cells for directly converting light energy into electricity. Significantly higher photocurrents have been reported for larger size CdS particles precipitated from solution in Nafion films 


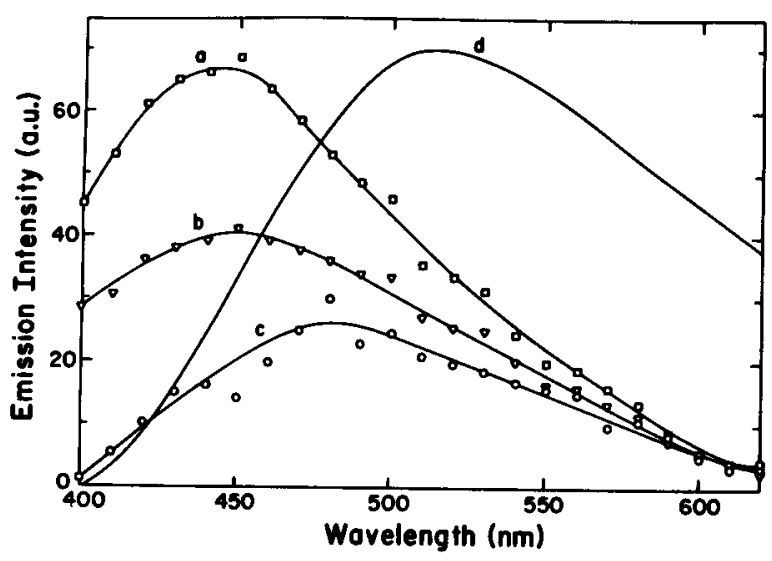

Figure 4. Time-resolved emission spectra of CdS clusters in Nafion film. The spectra were recorded following the excitation of the CdS doped Nafion film with $337 \mathrm{~nm}$ laser pulses: (a) $1.2 \mathrm{~ns}$; (b) $3.6 \mathrm{ns;}$ and (c) $52 \mathrm{~ns}$. The spectrum (d) is the steady state emission spectra at $77 \mathrm{~K}$ shown for comparison. (The intensity scale of spectrum (d) is not normalized with the scale for the spectra (a)-(c).)

(Honda et al 1988). We also checked this effect for quantized CdS particles by casting the film on a conducting glass electrode using Nafion solution (5\% in alcohol, Aldrich). The Nafion modified electrode was then doped with $\mathrm{Cd}^{2+}$ and exposed to an $\mathrm{H}_{2} \mathrm{~S}$ atmosphere. Unfortunately, the quantized CdS clusters prepared in this Nafion membrane exhibited very small photocurrents $(<100 \mathrm{nA})$. Since these small clusters are isolated, it is not possible to observe the collective photoelectrochemical effect as seen in particulate films directly cast on conducting glass plates (Hotchandani and Kamat 1992).

The quantized CdS clusters have potential applications in developing materials for nonlinear optics. CdS and CdSe clusters exhibit transient bleaching in the subnanosecond time domain (see, for example, Hilenski et al 1988, Kamat et al 1989, Gopidas and Kamat 1990). This transient bleaching, arising as a result of a photoinduced blue-shift in the absorption edge of the semiconductor, has been attributed to the decrease in the oscillator strength of the excitonic transitions in quantized clusters (Hilenski et al 1988) and dynamic Burstein-Moss effect in larger clusters (Kamat et al 1989). Time-resolved study of the transient bleaching of CdSe clusters in Nafion film has been reported in our earlier study (Gopidas and Kamat 1990).

\section{Conclusions}

CdS clusters which exhibit size quantization effect are prepared in Nafion film. The photophysical properties of these nanoclusters can be varied by controlling the growth of $\mathrm{CdS}$ in the polymer matrix. These semiconductor clusters are fluorescent with lifetimes ranging from $0 \cdot 85-480 \mathrm{~ns}$. The photoinduced charge-transfer processes have been probed by time-resolved emission spectroscopy. A better understanding of these photoinduced processes is essential before one could use these systems for solar energy conversion. 


\section{Acknowledgements}

The work described herein was supported by the Office of Basic Energy Sciences of the US Department of Energy. This is Contribution No. NDRL-3555 from the Notre Dame Radiation Laboratory.

\section{References}

Bard A J 1982 J. Phys. Chem. 86172

Bawendi M G, Steigerwald M L and Brus L E 1990 Annu. Rev. Phys. Chem. 41477

Brus L E 1986 J. Phys. Chem. 902555

Chestnoy N, Harris T D and Brus L E 1986 J. Phys. Chem. 903393

Dalas E, Sakkopoulos S, Kallitsis J, Vitoratos E and Koutsoukos P G 1990 Langmuir 61356

Federici J, Helman W P, Hug G L, Kane C and Patterson L K 1985 Comput. Chem. 9171

Fendler J H 1985 J. Phys. Chem. 892730

Fendler J H 1987 Chem. Rev. 87877

Finlayson M F, Park K H, Kakuta N, Bard A J, Campion A, Fox M A, Webber S E and White J M 1988 J. Luminesc. 39205

Fox M A 1991 Res. Chem. Intermed. 15153

Gopidas K R and Kamat P V 1990 Mater. Lett. 9372

Grätzel M 1989 Heterogeneous photochemical electron transfer (Boca Raton, FL: CRC Press) chap. 3

Henglein A 1988 Top Curr. Chem. 143113

Henglein A 1989 Chem. Rev. 891861

Hilenski E F, Lucas P A and Wang Y 1988 J. Phys. Chem. 893435

Honda K, Kuwano A, Chiba K, Iskikawa A and Miyama H 1988 Chem. Lett. 195

Hotchandani S and Kamat P V 1992 J. Phys. Chem. 966834

James D R, Liu Y-S, De Mayo P and Ware W R 1985 Chem. Phys. Lett. 120460

Kalyanasundaram K, Grätzel M and Pelizzetti E 1986 Coord. Chem. Rev. 6957

Kamat P V 1991 Kinetics and catalysis in microheterogeneous systems (eds) M Grätzel and K Kalyanasundaram (New York: Marcel Dekker) pp. 375-436

Kamat P V 1993 Chem. Rev. (in press)

Kamat P V and Dimitrijević N M 1990 Solar Energy 4483

Kamat P V, Dimitrijević N M and Nozik A J 1989 J. Phys. Chem. 932873

Kamat P V and Meisel D (eds) 1993 Isr. J. Chem. A special issue on Quantum Size Particles.

Krishnan M, White J R, Fox M A and Bard A J $1983 \mathrm{~J}$. Am. Chem. Soc. 1057002

Kuczynski B H, Milosavljevic B H and Thomas J K 1984 J. Phys. Chem. 88980

Mahler W 1988 Inorg. Chem. 27436

Meisner D, Memming R and Kastening 1983 Chem. Phys. Lett. 9634

Memming R 1988 Top. Curr. Chem. 14379

Misawa K, Yao H, Hayashi T and Kobayashi T 1991 Chem. Phys. Lett. 183113

Miyoshi H, Tanaka K, Uchida H, Yoneyama H, Mori H and Sakata T 1990a J. Electroanal. Chem. 29571

Miyoshi H, Yamachika M, Yoneyama H and Mori H 1990b J. Chem. Soc., Faraday Trans. 86815

Nagarajan V and Fessenden R W 1985 J. Phys. Chem. 892330

Nosaka Y, Yamaguchi K, Yokoyama H and Miyama H 1989 MRS International Meeting on Adv. Mater., (Pittsburgh: Materials Research Society) 12155

Smotkin E S, Brown R M, Rabenberg L K, Salmon K, Bard A J, Campion A, Fox M A, Mallouk T E, Webber S E and White J M 1990 J. Phys. Chem. 947543

Steigerwald M L and Brus L E 1990 Acc. Chem. Res. 23183

Wang Y and Herron N 1991 J. Phys. Chem. 95525

Wang Y and Mahler W 1987 Opt. Commun. 61233

Wilner I and Wilner B S 1988 Int. J. Hydrogen Energy 13593

Yoneyama H 1991 Res. Chem. Intermed. 15101 